\title{
Examining attitudes toward and ability to interact with an online personal health record: A case study in Saudi Arabia
}

\author{
Bandar Faisal Al-Mifgai ${ }^{1}$, Joseph Sharit*1, Arzu Onar-Thomas ${ }^{2}$, Shihab Asfour ${ }^{1}$ \\ ${ }^{1}$ Department of Industrial Engineering, University of Miami, United States \\ ${ }^{2}$ St. Jude Children's Research Hospital, United States
}

Received: June 26, 2019

Accepted: August 4, 2019

Online Published: August 15, 2019

DOI: $10.5430 / \mathrm{ijh} . v 6 \mathrm{n} 1 \mathrm{p} 1$

URL: https://doi.org/10.5430/ijh.v6n1p1

\begin{abstract}
Objective: This study examined the ability for adults from a developing country to use a personal health record (PHR) to perform health-management tasks. The effects of gender differences as well as differences in attitudes about using the internet to manage health prior to and after exposure to the PHR were also investigated.

Methods: A simulation of a PHR based on a well-established U.S. online patient portal was designed and tailored for this particular study population. Two hundred and three adults with a mean age of 40.9 years were recruited from various areas in Saudi Arabia and asked to perform seven common health-management tasks of varying degrees of difficulty. Their electronic health literacy and health numeracy, as well as their attitudes about using online health systems for managing their health prior to and following their interaction with the PHR, were assessed using questionnaires.

Results: After controlling for education, perceived health status, and comfort using the internet, electronic health literacy and health numeracy were still found to be significant predictors of participants' task performance, but only for the more challenging health-management tasks. No important differences based on gender were found. Exposure to the PHR significantly increased the acceptability of using the internet for managing their health.

Conclusions: The change in attitudes following interaction with the PHR suggests that many adults in this society could benefit from these electronic health systems, including females who, due to cultural considerations, may desire greater control in managing their health. However, the importance of electronic health literacy and health numeracy suggests the need for designs that minimize the impact of these factors for successful performance of health-management tasks.
\end{abstract}

Key Words: Personal health records, Health numeracy, Electronic health literacy, Gender, Health-management task performance, Public health policies

\section{INTRODUCTION}

Electronic patient portals, which are personal health records (PHRs) that are tethered to patients' electronic medical records, allow patients, through a website, to securely ac- cess their personal health information directly from electronic devices such as a computer tablet, laptop, or smartphone. Typically, PHRs also contain various interactive tools for helping patients manage their health-related activities, ${ }^{[1]}$

\footnotetext{
*Correspondence: Joseph Sharit; Email: jsharit@miami.edu; Address: Department of Industrial Engineering, University of Miami, 1251 Memorial Dr, Coral Gables, FL, 33146, United States. 
which could potentially lead to decreased healthcare utilization and improved chronic disease control through better care coordination and patient empowerment. ${ }^{[2]}$

Although there has been considerable success in a number of countries in planning and implementing electronic healthcare delivery systems, including PHRs, in many developing countries, for example in Saudi Arabia, electronic healthcare systems have not yet been widely implemented. ${ }^{[3]}$ Even in the United States, many factors have been found to adversely affect the ability for people to use a PHR. ${ }^{[4]}$ Two important factors are users' health literacy and health numeracy skills, with eHealth literacy gaining more attention as managing health becomes more dependent on negotiating online information. More formally, eHealth literacy refers to the ability to find, understand, and apply knowledge from digital sources to address a health problem, ${ }^{[5,6]}$ whereas health numeracy has been defined as "the degree to which individuals have the capacity to access, process, interpret, communicate, and act on numerical, quantitative, graphical, biostatistical, and probabilistic health information needed to make effective health decisions."[7]

The impact of numeracy ability was demonstrated in previous research in which a simulated PHR, based on Epic's MyChart, was used to examine performance on 15 healthmanagement tasks by a diverse sample of adults. ${ }^{[8]}$ Numeracy ability was found to be a significant predictor of task performance even after accounting for participants' education, internet experience, and cognitive abilities, but only for the group of problems designated as more numerically complex. For example, among the tasks with the lowest completion rates was one which required interpreting the correct insulin dose schedule. This led these investigators to recommend considering audio or video explanations to help patients understand and interpret numeric information. ${ }^{[9]} \mathrm{Nu}-$ meracy ability was also found to be an important variable in a comprehensive usability study of the MyHealtheVet PHR designed for veterans in the U.S. ${ }^{[10]}$

A potentially critical factor that has been given very little consideration in studies that have examined user interaction with PHRs is the cultural context, which is considered important for the successful implementation of many systems, ${ }^{[11]}$ including health systems. ${ }^{[12]}$ For example, in Saudi Arabia, few hospitals have successfully involved their patients in their healthcare systems using interactive health-management tools such as PHRs. While they have acknowledged some of the culturally-related difficulties in implementing electronic health services, ${ }^{[13]}$ potentially critical factors such as electronic health literacy and health numeracy have not been considered.
This article reports on a study that examined the ability for a sample of adults within Saudi Arabia to interact with a simulated PHR to perform common healthcare management tasks. Data were collected prior to the exposure to the PHR, during exposure to the PHR, and post exposure. First, we assessed the study participants' attitudes toward using the internet for managing their health prior to their interaction with the PHR, as well as their electronic health literacy and health numeracy. Next, we assessed their performance when using the PHR on seven common health-management tasks spanning different degrees of complexity. Finally, we assessed participants' attitudes about using the internet for managing their health after interacting with the PHR, as well as their ratings of usefulness and usability of the PHR. The goal was to use the study findings to help inform PHR interface design strategies for overcoming barriers to the introduction of PHRs into this society, and for making these tools more accessible and usable for this and other adult populations with similar socioeconomic characteristics.

\section{Methods}

\subsection{Participants}

The study participants consisted of a convenience sample of Saudi citizens. Participants were required to be at least 18 years of age, unfamiliar with using a PHR, not pregnant, and capable of providing written consent. They were recruited from areas such as shopping centers, coffee shops, universities, and hospitals within Riyadh, Mecca, the Eastern Province, and the adjacent rural towns and villages of Riyadh within Saudi Arabia (see Figure 1). Due to the culture and law in Saudi Arabia, the recruitment of female participants required that the male study investigator be accompanied by a female member from his own family while approaching potential female participants in public places. Of the 253 people who were approached, 203 voluntarily agreed to participate in the study without monetary compensation.

\subsection{PHR design}

A simulation of a PHR, which was referred to as MHSA (My Health Saudi Arabia), was developed and designed to be personalized to the Saudi adult population. This PHR's tools and the health-management tasks that could be performed were modeled after the MyHealtheVet patient portal designed for veterans in the U.S. ${ }^{[14]}$ The PHR was implemented on a website which participants logged into; this website also contained all the self-administered questionnaires from which data were collected. A major challenge in building the PHR concerned the language, which for this population needed to be in Arabic. Consequently, all the data collection tools and system interface were translated to Arabic and validated using Straker Translations Ltd., a certified translation company 
(www.strakertranslations.com).

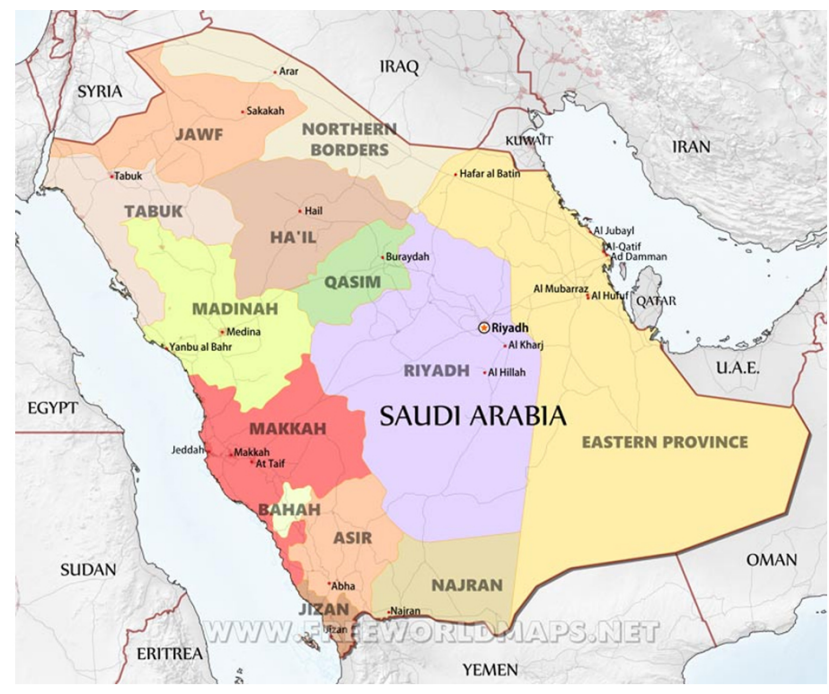

Figure 1. Map of Saudi Arabia showing the regions from where study participants were recruited, which included the Riyadh area, Mecca (Makkah on the map), and the Eastern Province

Source: Political Map of Saudi Arabia. Free World Maps, 30 July 2019, www.freeworldmaps.net/asia/saudiarabia/saudiarabia.jpg.

\subsection{Procedure}

After logging into the system, participants were directed to watch a two-minute introductory video which included a welcoming message and the detailed steps that needed to be followed on the website. Participants were then directed to complete three main sections: (1) the study questionnaires (demographic, occupational status, perceived health status, internet skills and use, acceptability of using the internet for managing health, eHealth literacy, and health numeracy); (2) the PHR performance tasks; and (3) the post-interaction questionnaires, which assessed the acceptability of using a PHR for managing health and the perceived usefulness and usability of the PHR. The demographic, occupational status, perceived health status, and internet skills and use questionnaires were adapted from the Qualtrics Survey Research Suite (Qualtrics, Provo, UT), translated to Arabic, and validated by Straker Translations Ltd.

Due to the cultural constraints, it was necessary that each participant perform the tasks at the location where the participant was recruited. However, as people can use a PHR at any place that has wi-fi service, this approach also served to mimic realistic situations in which people interact with their PHRs. To control for noise and environmental effects, all participants used the same laptop (Hewlett-Packard, Spectre), mouse, and noise-cancelling headphones for accessing the PHR website and completing the questionnaires and tasks.
The time duration of the study was four months. On average, participants were recruited for about an hour of their time.

\subsection{PHR health-management tasks}

Each participant was asked to complete seven tasks pertaining to a hypothetical, but realistic 76-year-old Saudi female named Norah. This approach to standardizing the assessment of performance across all study participants was consistent with the approach used in previous research involving assessing task performance using PHRs. ${ }^{[8,10]}$ As some of the tasks had several subtasks, the following coding scheme was used to compute a performance score for each task: a score of two was assigned if all subtasks (or the task, if there were no subtasks) were completed correctly; a score of one was assigned if at least one subtask (or if the single task) was performed partially correct); and a score of zero was assigned if the task was skipped or performed incorrectly. ${ }^{[8]}$ A random sample of 50 participants was used to validate the assignment of these scores to the seven tasks, with complete agreement found between the scores assigned by the experimenter and the scores assigned by an independent rater from the study team.

Prior to data collection, the level of difficulty of each of the seven tasks was evaluated independently by six raters, including three healthcare professionals, on a 5-point Likert scale: $1=$ very easy, $5=$ very difficult (see Table 1 ). The mean of these six ratings was computed for each task, and these seven mean difficulty levels were then used as weights to compute a weighted total performance score for each participant (in addition to calculating the unweighted total task performance scores). Tasks which scored 3 or higher were classified as "harder" and those below 3 were classified as "simpler."

Figures 2-4 show examples of the screens associated with tasks 1,3 , and 4 , respectively. When performing any of the tasks the participant had the opportunity to view a corresponding help video, which was indicated in the upper right-hand corner of the display window. Three study team members independently assessed the design of each of the screen interfaces, including the help videos, for usability concerns, and based on these assessments changes were iteratively introduced.

\subsection{Measures}

Demographic data were collected on age, gender, level of education, employment status, and monthly income. As many participants were unwilling to reveal income, these data were not used in subsequent analyses.

Electronic health (eHealth) literacy (eHEALS) was measured using the 8-question eHealth literacy scale. ${ }^{[6]}$ Responses range from 0 to 4 (maximum score $=32$ ). Health numeracy 
was measured using the 6-item General Health Numeracy scale (GHNT-6). ${ }^{[7]}$ Responses on each item are scored as either correct (1) or incorrect (0); thus the maximum score is six.

Perceived health status was assessed on a 5-point Likert scale: good physical health (e.g., no significant illnesses or disabilities); mildly physically impaired; moderately physically impaired; severely physically impaired; and totally physically impaired (e.g., requiring full-time medical assistance or nursing care). There were no participants in either of the two latter categories; thus, this variable was recoded to include only the first three categories.

Table 1. The seven health management tasks performed on the simulated PHR, the corresponding number of subtasks (shown in parentheses), and their task difficulty weights

\begin{tabular}{ll}
\hline Task & Weight \\
\hline 1. Schedule a routine lab test appointment at a particular place and time (9) & 1.83 \\
2. Medication management task: select 3 medications for refill (4) & 2.17 \\
3. Perform the action for managing a missed dosage (6) & 3.00 \\
4. Use the lab results report to determine the patient's glucose level (1) & 3.67 \\
5. Use a blood pressure (BP) chart to determine the patient's systolic BP (1) & 4.50 \\
6. Determine health history related to stomach conditions (1) & 1.50 \\
7. Determine allergies related to medications for the patient (1) & 1.83 \\
\hline
\end{tabular}

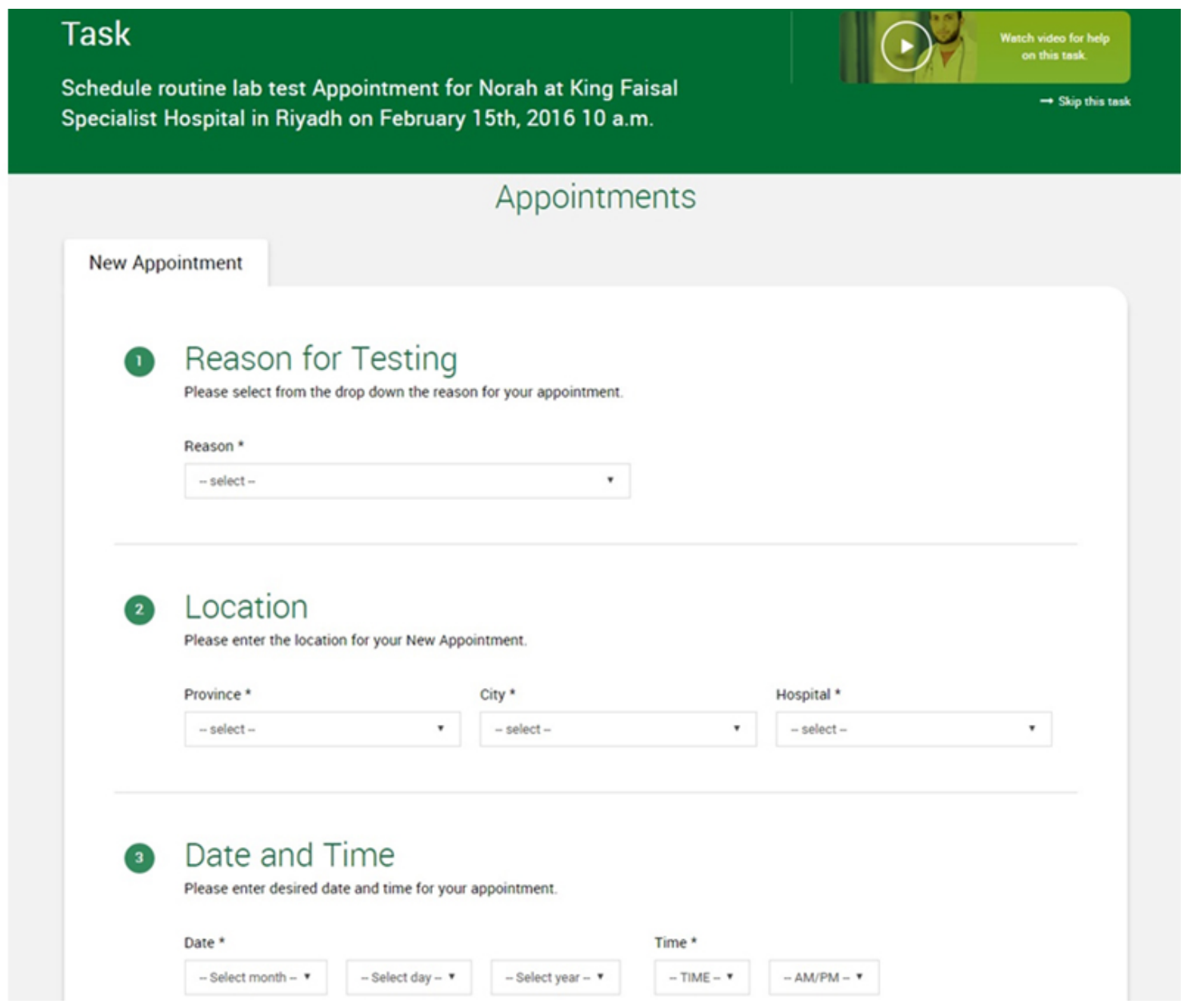

Figure 2. The display for the lab test appointment task (task 1) 


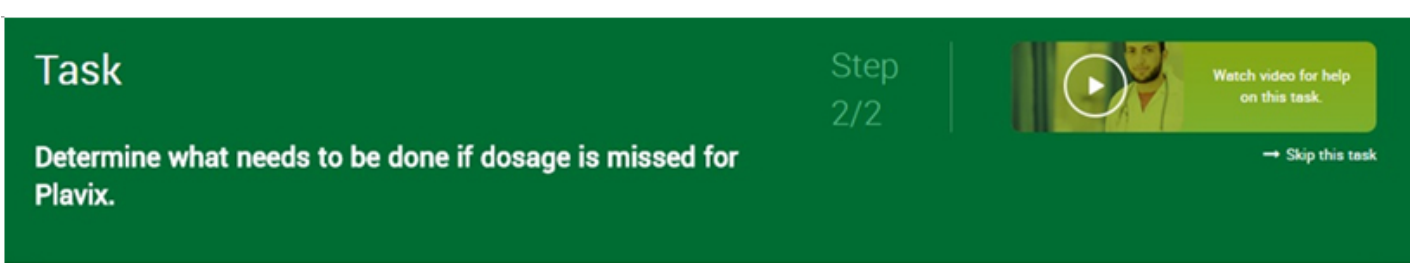

Please click on the title of each medication to see instructions if dosage is missed.

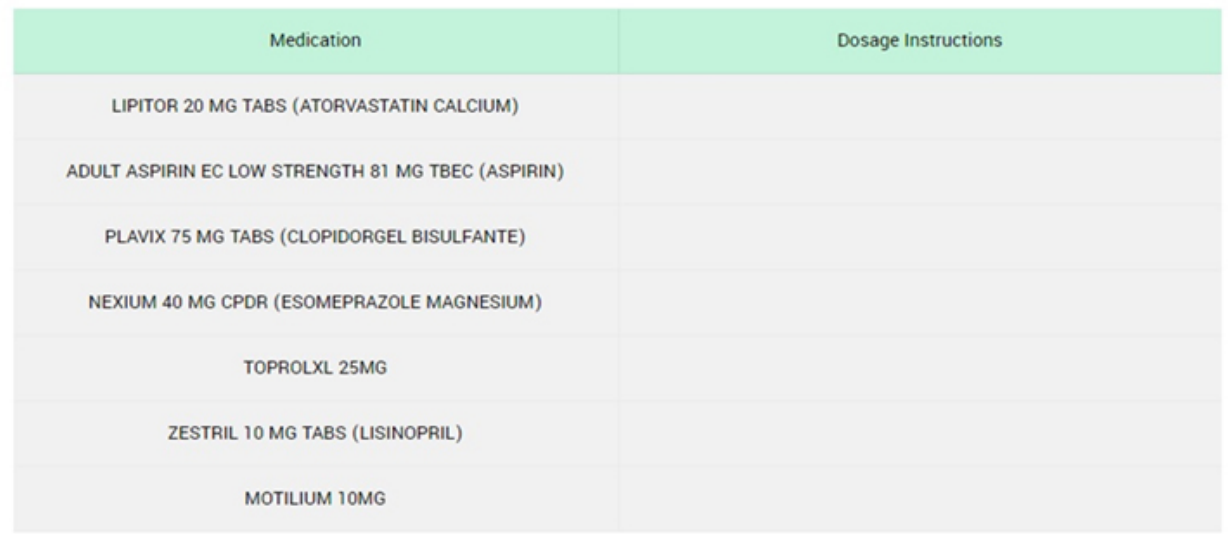

Norah doesn't remember if she toke her Plavix medication, what should she do?

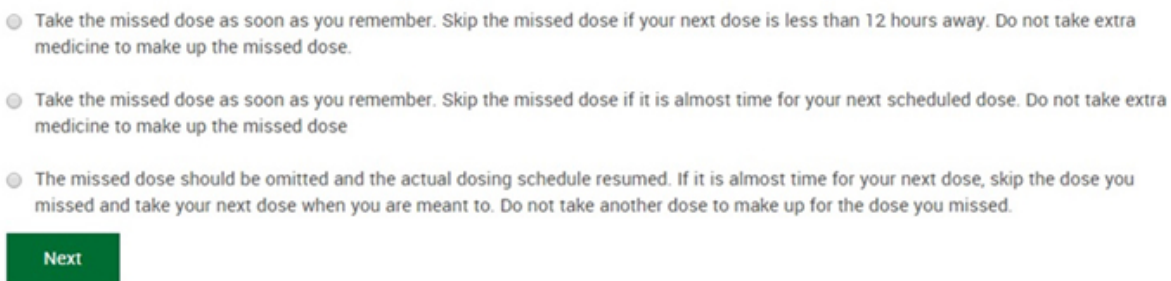

Figure 3. The display for the missed dosage task (task 3)

Perceived comfort using the internet was assessed through two questions: How comfortable do you feel using the internet? ( 0 = very uncomfortable, 4 = very comfortable), and How satisfied are you with your current skills for using the internet? ( $0=$ very unsatisfied, $4=$ very satisfied $)$.

To assess task performance on the health-management tasks, each participant's total (unweighted) task performance score was computed by summing the seven individual (unweighted) task scores. Each participant's weighted task performance score was computed by multiplying the product of the score for each task $(0,1$, or 2$)$ by its corresponding difficulty weight (see Table 1) and then summing the individual weighted scores across the seven tasks. Participants' task performance scores were also computed for the simpler and harder tasks separately by computing the sum of the four simpler task performance scores and the sum of the three harder task performance scores, respectively.

As the PHR enabled, within the page associated with each

Published by Sciedu Press task, the opportunity for participants to view a help video to aid them in completing the task, data on use of this video were also collected. The measure of use of this video aid was binary: either they used the video at least once or never used the video.

Attitudes about using the internet to manage health were measured as follows. Prior to participants' interaction with the PHR they were administered the following question: In general how comfortable do you feel using the internet for managing your health (set up appointments, manage medications, and view lab results)? They were then administered the following question after interacting with the PHR: In general how comfortable would you feel using a patient portal such as the one you just experienced for managing your health? Responses to these questions ranged from 4 (very comfortable) to 0 (very uncomfortable). The difference between preand post-exposure scores was coded as a nominal variable at three levels: acceptability in using the internet to manage health decreased, increased, or did not change. 


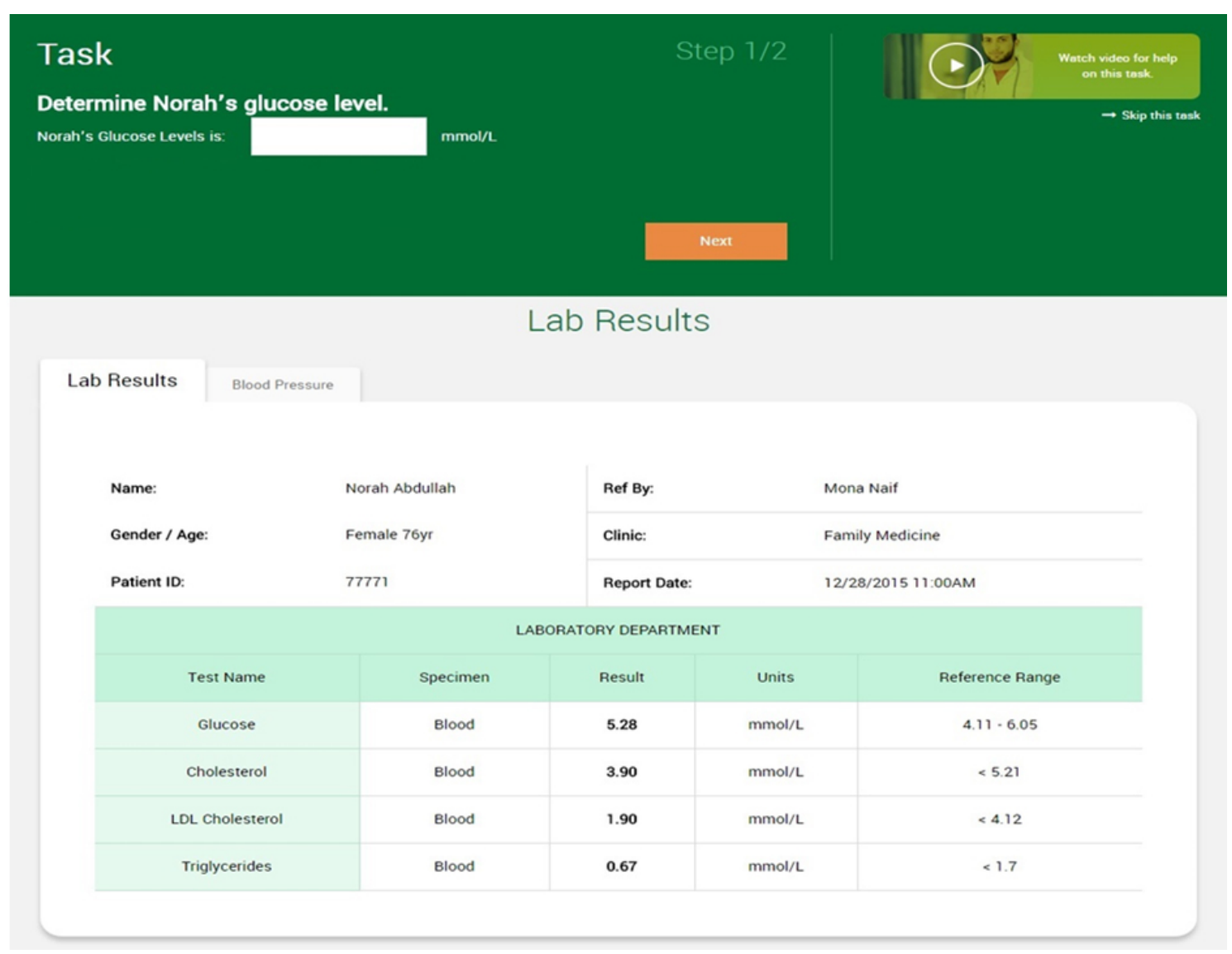

Figure 4. The display for the glucose level determination task (task 4)

Perceived usefulness and usability of the PHR were assessed from a questionnaire adapted from a usability study on $\mathrm{My}$ HealtheVet. ${ }^{[10]}$ The measure of perceived usefulness was computed using five items; 3 positive items (e.g., Having an option available such as this PHR could improve my ability to manage my health), and 2 negative items (e.g., I would feel much more comfortable speaking directly with my doctors or the medical staff about my health than using such a PHR to get health information). The measure of perceived usability was computed using 8 items; 3 positive items (e.g., I think using this PHR would be a fun experience), and 5 negative items (e.g., I found it easy to get lost when navigating around this PHR). Responses on each positive item ranged from 4 (strongly agree) to 0 (strongly disagree); responses on negative items were reverse coded.

\subsection{Statistical analysis}

Measures reflecting scores (e.g., task performance) or continuous measurements (e.g., age) were summarized using means (M) and standard deviations (SD). Categorical or Likert-scale measures (i.e., measures based on ordinal scales which assess levels of agreement or disagreement) were summarized using proportions except where composite scores from multiple questions are reported (e.g., for perceived usefulness and usability). Independent sample t-tests were used to compare two means and chi-square tests were used to compare proportions where appropriate. Ordinal logistic regression with logit function was used to examine potential predictors of acceptability of using the internet for managing health prior to interaction with the PHR. Nonparametric Mann-Whitney $U$ tests were used to test for differences in gender on measures of perceived comfort using the internet and perceived health status. Hierarchical regressions were used to test the hypotheses that eHealth literacy and health numeracy would be significant predictors of total task performance scores, as well as task performance scores for the simpler and harder tasks, even after controlling for education, perceived health status, and perceived comfort using the internet. For all analyses in this study, the significance level was set to $p<.05$. Analyses were performed using SPSS v.21.0.

\subsection{Ethical considerations}

This study was approved by the Institutional Review Board of the university in the U.S. that was responsible for the design and execution of the study, and by the government of Saudi Arabia. All participants were informed that their 
participation was voluntary and that they could withdraw from the study at any time and without indicating reasons. A written consent was obtained from all participants before the collection and use of the data. Data were kept confidential and inaccessible to anybody but to the research team.

\section{RESULTS}

\subsection{Demographics}

The ages of the 203 participants ranged from 18 to 75 years $(\mathrm{M}=40.9, \mathrm{SD}=17.1)$, and 59\% were female. One-hundred and sixty-four of the participants resided in urban areas whereas 39 lived in rural areas. The native spoken language for all the participants was Arabic. One hundred and nineteen $(58.6 \%)$ of the participants were employed, 42 (20.7\%) were unemployed, $18(8.9 \%)$ were retired, and 24 (11.8\%) were students. Twenty-eight $(13.8 \%)$ of the participants had less than a high school level of education, $33(16.3 \%)$ had a high school or equivalent degree, 35 (17.2\%) had some college or vocational school, 70 (34.5\%) had a college degree, and $37(18.2 \%)$ had post-graduate or professional degrees. These data indicate that participants in this study were overall better educated compared to the general Saudi population where, in comparison, $40.2 \%$ of the population has less than a high school level of education (based on 2015 Saudi Census data).

\subsection{Electronic health literacy and general health numer-} acy scores

The participants' eHealth literacy scores (eHEALS) ranged from $0-32$, with $\mathrm{M}=15.4$ and $\mathrm{SD}=9.5$. The participants' health numeracy scores (GHNT-6) ranged from 0 to 6 , with $\mathrm{M}=2.5$ and $\mathrm{SD}=1.6$. Table 2 shows how scores differed on these two measures based on gender, geographical location (urban versus rural), and education level (less than college versus at least college).

Table 2. Comparisons of means and standard deviations for eHEALS and GHNT-6 based on gender, geographical location, and education

\begin{tabular}{llllllll}
\hline \multicolumn{7}{c}{ eHEALS } \\
\hline $\begin{array}{l}\text { Males } \\
\text { Urban }\end{array}$ & $\mathrm{M}=16.15$ & $\mathrm{SD}=9.40$ & $(\mathrm{n}=84)$ & Females & $\mathrm{M}=14.89$ & $\mathrm{SD}=9.56$ & $(\mathrm{n}=119)$ \\
$<$ College & $\mathrm{M}=16.24$ & $\mathrm{SD}=9.12$ & $(\mathrm{n}=164)$ & Rural & $\mathrm{M}=11.95$ & $\mathrm{SD}=10.33$ & $(\mathrm{n}=39)$ \\
& & $\mathrm{SD}=7.95$ & $(\mathrm{n}=61)$ & $\geq$ College & $\mathrm{M}=19.20$ & $\mathrm{SD}=7.34$ & $(\mathrm{n}=142)$ \\
\hline Males & $\mathrm{M}=2.14$ & $\mathrm{SD}=1.44$ & $(\mathrm{n}=84)$ & FHNT-6 & & \\
Urban & $\mathrm{M}=2.60$ & $\mathrm{SD}=1.57$ & $(\mathrm{n}=164)$ & Rural & $\mathrm{M}=2.03$ & $\mathrm{SD}=1.71$ & $(\mathrm{n}=39)$ \\
$<$ College & $\mathrm{M}=1.48$ & $\mathrm{SD}=1.23$ & $(\mathrm{n}=61)$ & $\geq$ College & $\mathrm{M}=2.93$ & $\mathrm{SD}=1.56$ & $(\mathrm{n}=142)$ \\
\hline
\end{tabular}

\subsection{Perceived comfort using the internet and perceived health status}

About three fourths of the study participants either had a desktop or a laptop. In addition, $88.2 \%$ reported having access to a smartphone. About $70 \%$ of the participants reported feeling comfortable using the internet, and $71.4 \%$ reported feeling satisfied with their internet navigation skills. Most of the study participants perceived their overall health status as good $(77.3 \%), 18.7 \%$ reported that they were mildly physically impaired, and $3.9 \%$ reported that they were moderately physically impaired.

\subsection{Acceptability of using the internet for managing health information}

Prior to their exposure to the PHR, $48.2 \%$ of the participants indicated that they felt either very uncomfortable or somewhat uncomfortable using the internet for managing their health, and $32(15.8 \%)$ reported feeling neither comfortable nor uncomfortable. In contrast, 31 (15.3\%), and 42 (20.7\%) participants felt somewhat comfortable and very comfortable, respectively. To examine predictors of this acceptability, ordinal logistic regression analyses multiple testing models were used that included age, gender, education, eHealth literacy, health numeracy, perceived health status, and perceived comfort using the internet as the predictor variables. The only variable found to be predictive of this acceptability measure was perceived comfort using the internet. For a one unit increase in perceived comfort using the internet (i.e., going from one level to the next on this questionnaire item), there was a 2.17 -unit increase $(p<.001)$ in the ordered log odds of being in a higher level of acceptability of using the internet for managing health. The model with the best goodness-of-fit test was the one that only included perceived comfort using the internet (Cox and Snell adjusted $\mathrm{R}^{2}=.56, p<.001$ ).

\subsection{PHR task performance}

The three measures of task performance reported are the total weighted task performance scores, the task performance scores for the simpler tasks, and the task performance scores for the harder tasks. The results for the unweighted total task 
performance scores were very consistent with those for the weighted scores; the latter, however, provided additional statistical significance, likely due to this measure having greater resolution.

For the total weighted task performance score measure, the first model in hierarchical regression analysis indicated that all three control variables (education, perceived health status, and perceived comfort using the internet) were significant predictors of task performance (see Table 3). Following the addition of the eHealth literacy and health numeracy variables in step 2, both measures were found to significantly predict task performance; however, while perceived health status and perceived comfort using the internet remained significant predictors in step 2 , education no longer was.

Table 3. Hierarchical regression analysis for total (weighted) task performance scores

\begin{tabular}{llll}
\hline Model Steps & Beta $^{\#}$ & t & p-value \\
\hline Model 1 & & & \\
(Constant) & & 14.12 & $<.001$ \\
Education level & .116 & 2.04 & .042 \\
Perceived health status & .357 & 6.22 & $<.001$ \\
Perceived comfort using the internet & .419 & 6.59 & $<.001$ \\
Model 2 & & & \\
(Constant) & & 14.74 & $<.001$ \\
Education level & .090 & 1.63 & .104 \\
Perceived health status & .296 & 5.11 & $<.001$ \\
Perceived comfort using the internet & .245 & 2.95 & .004 \\
Electronic health literacy (eHEALS) & .176 & 2.22 & .027 \\
Health numeracy (GHNT-6) & .154 & 2.84 & .005 \\
\hline
\end{tabular}

Note. ${ }^{\text {\# }}$ Standardized coefficients

Table 4. Hierarchical regression analysis for task performance scores on the simpler tasks

\begin{tabular}{llll}
\hline Model Steps & Beta $^{\#}$ & t & p-value \\
\hline Model 1 & & & \\
(Constant) & & 41.10 & $<.001$ \\
Education level & .137 & 1.72 & .087 \\
Perceived health status & .420 & 5.19 & $<.001$ \\
Perceived comfort using the internet & -.108 & -1.21 & .228 \\
Model 2 & & & \\
(Constant) & & 40.67 & $<.001$ \\
Education level & .128 & 1.59 & .114 \\
Perceived health status & .396 & 4.69 & $<.001$ \\
Perceived comfort using the internet & -.160 & -1.33 & .187 \\
Electronic health literacy (eHEALS) & .031 & 0.27 & .789 \\
Health numeracy (GHNT-6) & .086 & 1.10 & .273 \\
\hline Note. ${ }^{\#}$ Standardized coefficients & & & \\
\hline
\end{tabular}

For the simpler tasks (see Table 4), following step 1 only perceived health status significantly predicted task performance.
Adding eHealth literacy and health numeracy resulted in a final model (step 2) in which perceived health status remained as the only significant predictor.

In contrast, analysis of performance of the harder tasks indicated that both eHealth literacy and health numeracy were significant predictors of performance, even after accounting for education, perceived health status, and perceived comfort using the internet (see Table 5).

Table 5. Hierarchical regression analysis for task performance scores on the harder tasks

\begin{tabular}{lccc}
\hline Model Steps & Beta $^{\#}$ & t & p-value \\
\hline Model 1 & & & \\
(Constant) & & 3.95 & $<.001$ \\
Education level & .100 & 1.78 & .077 \\
Perceived health status & .308 & 5.41 & $<.001$ \\
Perceived comfort using the Internet & .481 & 7.64 & $<.001$ \\
Model 2 & & & \\
(Constant) & & 4.38 & $<.001$ \\
Education level & .074 & 1.34 & .181 \\
Perceived health status & .246 & 4.28 & $<.001$ \\
Perceived comfort using the internet & .300 & 3.66 & $<.001$ \\
Electronic health literacy (eHEALS) & .186 & 2.39 & .018 \\
Health numeracy (GHNT-6) & .151 & 2.83 & .005 \\
\hline
\end{tabular}

Note. ${ }^{\#}$ Standardized coefficients

\subsection{Gender differences}

Independent $t$-tests revealed that males had significantly higher performance scores than females on the simpler tasks $(\mathrm{t}(198)=1.27, p=.026)$, although the differences in mean scores were marginal $(\mathrm{M}=14.2, \mathrm{SD}=0.9$ for males; $\mathrm{M}$ $=14.0, \mathrm{SD}=1.6$ for females). There were no significant gender differences in total task performance scores or for the harder tasks. In addition, no significant gender differences were found on eHealth literacy (males: $\mathrm{M}=16.2, \mathrm{SD}=9.4$; females: $M=14.9, \mathrm{SD}=9.6$ ). However, a significant effect for gender was found for health numeracy, $(\mathrm{t}(198)=2.6, p$ $=.012)$, with females $(\mathrm{M}=2.7, \mathrm{SD}=1.7)$ demonstrating higher scores than males $(\mathrm{M}=2.1, \mathrm{SD}=1.4)$. The results of Mann-Whitney $U$ tests indicated that there were no significant gender differences in either perceived comfort using the internet $(p=.281)$ or in perceived health status $(p=.781)$.

\subsection{Use of the help video}

As compared to participants who used the video at least once, those who did not had significantly higher mean eHealth literacy scores (20.88 as compared to 9.44) and significantly higher mean health numeracy scores (3.16 as compared to 1.76), based on independent $t$-tests ( $p<.001$ in both cases). Findings from a multiple binary logistic regression analysis that included gender, age, education, eHealth literacy, and 
health numeracy indicated that watching the video was positively associated with age: a one-year increase in age was significantly associated with a $7 \%$ increase in probability of watching the help video, Odds Ratio $(\mathrm{OR})=1.07 ; 95 \%$ confidence interval $(\mathrm{CI})=(1.04,1.11), p<.001$. In addition, there was an inverse relationship between eHealth literacy level and use of the help video: a $1 \%$ increase in eHealth literacy was associated with a $7 \%$ decrease in using help video, $\mathrm{OR}=0.93 ; 95 \% \mathrm{CI}=(0.874,0.990)$.

\subsection{Change in acceptability based on exposure to the PHR}

The three levels of change in acceptability were crossed with the categories associated with gender, level of education, and perceived health status in a series of chi-square analyses. Findings showed no significant differences in change in acceptability based on gender. However, differences in acceptability were found based on education level, $\chi(8)=19.17, p$ $=.014$, and level of perceived health status, $\chi(4)=9.92, p=$ .042. For education, acceptability increased across the lower education levels. For perceived health status, 100 out of the 157 participants who perceived their physical health as good reported higher levels of acceptability of using the internet for managing health.

\subsection{Perceived usefulness and usability of the PHR}

For the overall study sample, the mean (composite) usefulness score was $2.80(\mathrm{SD}=0.56)$ and the mean (composite) usability score was $2.92(\mathrm{SD}=0.75)$. Independent $t$-tests indicated no significant differences between males and females in either perceived usefulness or perceived usability scores. Notably, the perceived usefulness of the PHR was significantly positively correlated with the change in acceptability of using the internet for managing health (Spearman's rho $=0.164, p=.020$ ).

\section{Discussion}

A challenge that many developing countries face is establishing health-management systems that are more congruent with the movement toward digitization of health information. PHRs offer patients greater opportunity for self-management and empowerment of their health, and could be especially beneficial in societies where travel to medical facilities is difficult or where cultural norms dictate modesty in physicianpatient interactions.

In this study, we developed a PHR and tested it within such a society - specifically, on adult citizens within Saudi Arabia. We were interested in assessing the capabilities for these adults to perform simpler as well as harder healthmanagement tasks using this PHR, and in determining if

Published by Sciedu Press acceptability of using a PHR might change by virtue of exposure to this e-Health tool. Because health literacy and numeracy ability have been shown to be important predictors in other studies of PHR task performance in the U.S., ${ }^{[8,10]}$ emphasis was given in this study to the roles of electronic health (eHealth) literacy and health numeracy. These variables are potentially more relevant to electronic health tools such as PHRs ${ }^{[15]}$ and to our knowledge have not been previously investigated in developing countries such as Saudi Arabia.

This type of eHealth tool can be particularly beneficial for females in modest cultural societies such as Saudi Arabia due to their ability for enabling many health-related activities to be performed in private. ${ }^{[16]}$ On a positive note, the results indicated that gender should not be a concern for use of PHRs in this society, as gender was not associated with differences of statistical or practical significance in either task performance, initial attitudes in using the internet for managing health, changes in such attitudes based on exposure to the PHR, eHealth literacy or health numeracy skills, or perceived usefulness and usability of the PHR.

Importantly, eHealth literacy and health numeracy were found to be significant predictors of performance on the relatively harder health-management tasks, even after accounting for education level, perceived health status, and perceived comfort using the internet. These findings suggest that even marginal increases in the health task demands may have a large impact on the ability for this population of adults, and perhaps other users, to use these types of electronic health tools effectively. Thus, policy makers within this and similar societies should be concerned about the roles of eHealth literacy and health numeracy for successful diffusion of this technology. One strategy they might consider is to promote the use of other electronic health resources, such as the internet, for finding health information as this could enable relevant skills to translate into more successful use of interactive tools such as PHRs.

It is also important to consider ways, within such PHRs, to enable better comprehension of numeric health information. The fact that participants in this study who had lower health numeracy were more disposed toward using the help video indicates that such aids embedded within these PHRs would likely be used by those most in need, and thus should be geared, at least in part, toward understanding and managing numeric health information. ${ }^{[17]}$

\section{Limitations}

Several limitations with the study should be noted. First, because a convenience sample was used the findings from 
this study may not be generalizable to the entire population in Saudi Arabia. Second, this study intentionally focused on patient-related (i.e., individual) factors. Future research could investigate task performance on PHRs in the light of a broad theoretical framework (e.g., social ecological theory) that includes a wide range of individual and social factors. ${ }^{[18]}$ Also, research on the acceptability of using such e-Health systems could benefit by being guided by consumer theoretical models such as the Unified Theory of Acceptance and Use of Technology. ${ }^{[19-22]}$

\section{Conclusions}

In a study performed in Saudi Arabia, where uptake of interactive health-management systems such as electronic personal health records is low, electronic health literacy and health numeracy were found to be important predictors of successful performance on harder health-management tasks, even after controlling for other potentially important variables. Also the change in acceptability of using the internet for managing health following exposure to the PHR in this study was significantly correlated with the perceived usefulness of the system. There were no important gender differences in any of the study measures. Policy makers within this society may benefit from these findings by being more vigilant about the roles that eHealth literacy and health numeracy may play for a successful diffusion of this technology and by promoting the usefulness of this technology for managing health to improve its acceptability.

\section{ACKNOWLEDGEMENTS}

We thank the study participants for their willingness and patience in taking the time in public areas to participate in this study.

\section{FUNDING}

This study was funded in part by the Department of Industrial Engineering at the University of Miami, FL, USA.

\section{CONFlicts OF INTEREST Disclosure}

The authors declared no conflicts of interest with any of this research work, authorship, and/or publication.

\section{REFERENCES}

[1] Wynia M, Dunn K. Dreams and nightmares: practical and ethical issues for patients and physicians using personal health records. The Journal of Law, Medicine \& Ethics. 2010; 38(1): 64-73. PMid:20446985. https ://doi.org/10.1111/j.1748-720X . 20 $10.00467 . \mathrm{x}$

[2] Ralston JD, Hirsch IB, Hoath J, et al. Web-based collaborative care for type 2 diabetes: a pilot randomized trial. Diabetes Care. 2009; 32(2): 234-9. PMid:19017773. https://doi.org/10.2337/dc $08-1220$

[3] Ahmadi M, Jeddi FR, Gohari MR, et al. A Review of the Personal Health Records in Selected Countries and Iran. J Med Syst. 2012; 36(2): 371-82. PMid:20703713. https://doi.org/10.1007/s1 0916-010-9482-3

[4] Ozok AA, Wu H, Gurses AP. Exploring Patients' Use Intention of Personal Health Record Systems: Implications for Design. International Journal of Human-Computer Interaction. 2017; 33(4): 265-79. https://doi.org/10.1080/10447318.2016.1277637

[5] Alpert JM, Desens L, Krist AH, et al. Measuring Health Literacy Levels of a Patient Portal Using the CDC's Clear Communication Index. Health Promotion Practice. 2017; 18(1): 140-9. PMid:27188894. https://doi.org/10.1177/1524839916643703

[6] Norman DC, Skinner AH. eHEALS: The eHealth Literacy Scale. J of Medical Internet Research. 2006; 8(4): e27. PMid:17213046. https://doi.org/10.2196/jmir.8.4.e27

[7] Golbeck AL, Ahlers-Schmidt CR, Paschal AM, et al. A definition and operational framework for health numeracy. Am J of Preventive Medicine. 2005; 29(4): 375-6. PMid:16242604. https: //doi.org/10.1016/j.amepre.2005.06.012

[8] Taha J, Czaja SJ, Sharit J, et al. Factors affecting usage of a personal health record (PHR) to manage health. Psychology and Aging. 2013;
28(4): 1124-39. PMid:24364414. https : //doi .org/10.1037/a0 033911

[9] Taha J, Sharit J, Czaja SJ. Usability of an Electronic Personal Health Record (PHR) Among a Diverse Group of Adults. Proceedings of the Human Factors and Ergonomics Society Annual Meeting. 2014; 58(1): 619-23. https://doi.org/10.1177/1541931214 581132

[10] Sharit J, Lisigurski M, Andrade AD, et al. The roles of health literacy, numeracy, and graph literacy on the usability of the VA's personal health record by veterans. J Usability Studies. 2014; 9(4): 173-93.

[11] Moray N. Culture, politics and ergonomics. Ergonomics. 2000; 43(7): 858-68. PMid:10929822. https://doi .org/10.1080/00140130 0409062

[12] S. Valdez R, Brennan PF. Embracing Complexity: Rethinking Culturally Informed Design in Human Factors/Ergonomics and Consumer Health Informatics. International J of Human-Computer Interaction. 2017; 33(4): 322-32. https://doi.org/10.1080/10447318.2 016.1269429

[13] Alswailem ASaO. Connecting the hospital to the patient: Patient portals and mobile healthcare information and management systems society (HIMSS). Middle East Integrated Health Innovations Conference; Dubai, United Arab Emirates 2013.

[14] Nazi KM. Veterans' voices: use of the American Customer Satisfaction Index (ACSI) Survey to identify My HealtheVet personal health record users' characteristics, needs, and preferences. Journal of the American Medical Informatics Association: J of Am Med Informatics Association. 2010; 17(2): 203-11. PMid:20190065. https ://doi.org/10.1136/jamia.2009.000240

[15] Diviani N, van den Putte B, Giani S, et al. Low health literacy and evaluation of online health information: a systematic review of the literature. J of Medical Internet Research. 2015; 17(5). PMid:25953147. https://doi.org/10.2196/jmir.4018 
[16] AlGhamdi KM, Moussa NA. Internet use by the public to search for health-related information. International $\mathrm{J}$ of Medical Informatics. 2012; 81(6): 363-73. PMid:22217800. https://doi.org/10.101 $6 / j$. ijmedinf.2011.12.004

[17] Garcia-Retamero R, Andrade A, Sharit J, et al. Is Patients' Numeracy Related to Physical and Mental Health? Medical Decision Making. 2015; 35(4): 501-11. PMid:25943579. https ://doi .org/10.117 7/0272989X15578126

[18] Bronfenbrenner U. The experimental ecology of human development. Cambridge, MA: Harvard University Press; 1979.

[19] Chang IC, Hwang HG, Hung WF, et al. Physicians' acceptance of pharmacokinetics-based clinical decision support systems. Expert Systems with Applications. 2007; 33(2): 296-303. https: //doi.org/10.1016/j.eswa.2006.05.001
[20] Maillet É, Mathieu L, Sicotte C. Modeling factors explaining the acceptance, actual use and satisfaction of nurses using an Electronic Patient Record in acute care settings: An extension of the UTAUT. International J of Medical Informatics. 2015; 84(1): 36-47. PMid:25288192. https://doi.org/10.1016/j.ijmedinf . 201 4.09.004

[21] Vanneste D, Vermeulen B, Declercq A. Healthcare professionals' acceptance of BelRAI, a web-based system enabling person-centred recording and data sharing across care settings with interRAI instruments: a UTAUT analysis. BMC Medical Informatics and Decision Making. 2013; 13(1). PMid:24279650. https://doi.org/10.118 6/1472-6947-13-129

[22] Yi MY, Jackson JD, Park JS, et al. Understanding information technology acceptance by individual professionals: Toward an integrative view. Information \& Management. 2006; 43(3): 350-63. https://doi.org/10.1016/j.im.2005.08.006 